\title{
Dynamic Pattern Formation in a Vesicle-Generating Microfluidic Device
}

\author{
Todd Thorsen, ${ }^{1}$ Richard W. Roberts, ${ }^{1}$ Frances H. Arnold, ${ }^{1}$ and Stephen R. Quake ${ }^{2}$ \\ ${ }^{1}$ Division of Chemistry and Chemical Engineering, California Institute of Technology, Pasadena, California 91125 \\ ${ }^{2}$ Department of Applied Physics, California Institute of Technology, Pasadena, California 91125
}

(Received 9 January 2001)

\begin{abstract}
Spatiotemporal pattern formation occurs in a variety of nonequilibrium physical and chemical systems. Here we show that a microfluidic device designed to produce reverse micelles can generate complex, ordered patterns as it is continuously operated far from thermodynamic equilibrium. Flow in a microfluidic system is usually simple - viscous effects dominate and the low Reynolds number leads to laminar flow. Self-assembly of the vesicles into patterns depends on channel geometry and relative fluid pressures, enabling the production of motifs ranging from monodisperse droplets to helices and ribbons.
\end{abstract}

DOI: $10.1103 /$ PhysRevLett.86.4163

Complex pattern formation is ubiquitous in nature. Efforts to understand these effects have led to important insights into nonlinear dynamical systems and fundamental nonequilibrium physics [1]. Fluid systems have been fertile ground for pattern formation, with classic examples such as Rayleigh-Benard convection, Taylor-Couette flow in rotary systems, nonlinear surface waves, liquid crystals, and falling droplets [2]. Key ideas that have emerged from the study of pattern formation are the central roles of instability and nonlinearity, as well as the influence of perturbations and boundary conditions on the morphology of the patterns. The elements of instability and nonlinearity are generally not present in microfluidic devices because the length scales are small enough that inertial effects in the fluid can be neglected. As most microfluidic devices operate at low Reynolds number [3], the Navier-Stokes equation for fluid flow becomes linear and the flow is laminar. This result has many practical consequences for efforts to miniaturize biological assays and produce lab on a chip system $[4,5]$. In this Letter, we show how the interaction between two immiscible fluids can be used to introduce nonlinearity and instability in a microfluidic device. The resulting complex pattern formation is an unexpected and fascinating example of self-organization in a dynamic system far from equilibrium.

Emulsions are formed by shearing one liquid into a second immiscible one, often in the presence of a surfactant, to create small droplets. The droplets can be remarkably stable, maintaining their shape and distribution for years [6]. Significant advances have been made in the past few years to produce emulsions that are monodisperse, with standard deviations in droplet size less than 5\% [7-10]. Unlike the standard crossflow techniques for generating water-in-oil emulsions, in which the discontinuous phase is forced through narrow pores $[8,10]$ or capillaries $[7,11]$ into an open continuous phase, we accomplish droplet formation at the junction of two microfluidic channels containing water and an oil surfactant mixture, respectively. The water partially obstructs flow at the junction, but is not broken off at the channel interface as in traditional crossflow devices. Droplet formation is achieved by high shear
PACS numbers: 82.40.Ck, 47.54.+r, 61.30.Pq, 82.70.Uv

forces generated at the leading edge of the water perpendicular to the oil flow, generating picoliter-scale droplets. Although the system remains at low Reynolds number, the flow is nonlinear because of interactions on the boundary between the two fluids. The two important effects are that the boundary is not static and that the motion of one fluid can entrain the other [12]. The resulting instability that drives droplet formation is a well-known competition between surface tension and shear forces [13].

The emergence of static crystalline structure in emulsions has been documented previously [7,9]. In our experiments, the droplets self-assemble into a variety of coherent, moving patterns as they are formed. We examine the control parameters that lead to vesicle formation and organization in an emulsion in a microfluidic device, illustrating the relationship between droplet pattern formation, pressure, and the geometric boundary conditions of the system. The droplet size and frequency can be precisely controlled by modifying the relative pressure of water and oil, enabling the production of a wide range of vesicle shapes and patterns. Under conditions where the water pressure is lower than the oil pressure, monodisperse separated reverse micelles are formed. As the relative water pressure is increased at fixed oil pressure, the droplets become ordered into a single continuous stream. At water pressures that exceed the oil pressure, complex, organized patterns begin to emerge in the stream, ranging from helical-like structures to coherent ribbon motifs.

The microfluidic devices utilized in our experiments are fabricated by pouring acrylated urethane (Ebecryl 270, UCB Chemicals) on a silicon wafer mold containing positive-relief channels patterned in photoresist (SJR5740, Shipley), which is then cured by exposure to UV light. The channels are fully encapsulated by curing the patterned urethane on a coverslip coated with a thin layer of urethane and bonding the two layers together through an additional UV light exposure. The measured channel dimensions are approximately $60 \mu \mathrm{m}$ wide $\times 9 \mu \mathrm{m}$ high, tapering to $35 \mu \mathrm{m} \times 6.5 \mu \mathrm{m}$ in the region where the water and oil/surfactant mixture meet at the crossflow intersection (Fig. 1). Input and post-crossflow junction channel lengths 


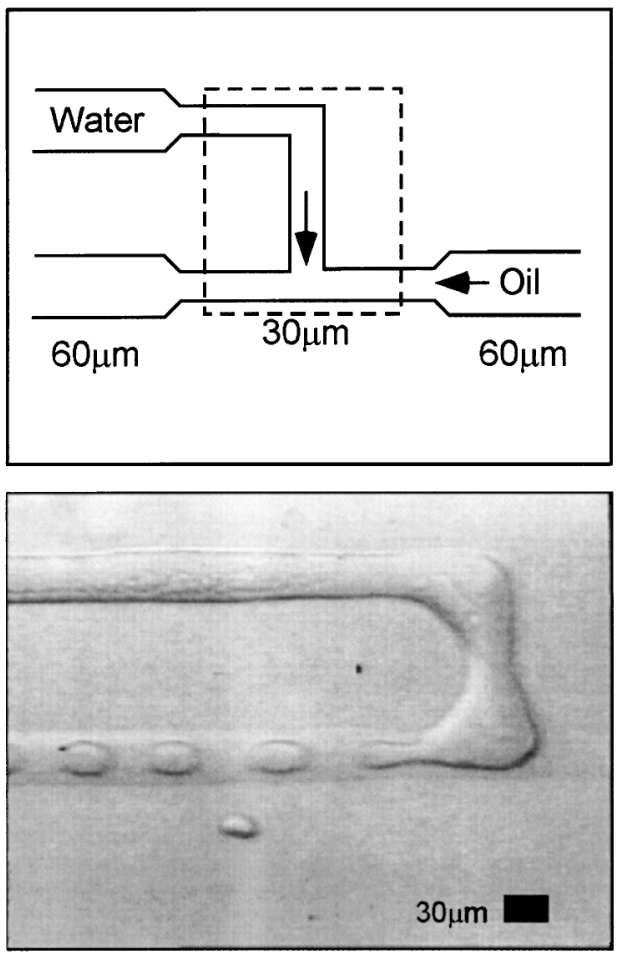

FIG. 1. Microfabricated channel dimensions at the point of crossflow and photomicrograph of the discontinuous water phase introduced into the continuous oil phase. Dashed rectangle indicates area in photomicrograph.

$(60 \mu \mathrm{m}$ wide $\times 9 \mu \mathrm{m})$ are $\sim 1$ and $\sim 4 \mathrm{~cm}$, respectively. The fluids are introduced into the urethane microfluidic devices through pressurized reservoirs containing water and oil. The reservoirs are connected to the device through approximately $30 \mathrm{~cm}$ of $500 \mu \mathrm{m}$ i.d. Tygon tubing. Pressure was applied to the reservoirs with compressed air, and the device output channel was allowed to vent to the atmosphere. All reported pressures are relative to atmospheric pressure (psig). Various oils were tested in the device, including decane, tetradecane, and hexadecane, combined with the surfactant Span 80 concentrations $(v / v)$ of $0.5 \%, 1.0 \%$, and $2 \%$. The device is equilibrated prior to crossflow by priming the outflow channel with oil/surfactant to eliminate water interaction with the hydrophilic urethane. The production of reverse micelles is then initiated by modifying relative oil/surfactant and water pressures such that the water enters the crossflow junction perpendicular to the oil stream, shearing off into discrete droplets (Fig. 1).

The shape of the channels influences the size distribution and morphology of the droplet patterning and can be modified by heating the photoresist mold on the silicon wafer $\left(80-110^{\circ} \mathrm{C}\right)$ to round the normally rectangular channels. The photoresist flows during the heating process, creating localized maxima and minima at the perpendicular intersection in the mold where the water is sheared into the oil/surfactant phase and the transitions from the restricted to the wide channels. Channels that have not been rounded produce only monodisperse reverse micelles with regular periodicity that associate with the walls of the wide channel as they flow through the device (Fig. 2). The relative water/oil-surfactant pressures determine the size and spacing between the reverse micelles. The patterns in a rounded channel are more complex, ranging from periodic droplets to "ribbons," "pearl necklaces," and helical intermediate structures. The self-organization of the reverse micelles depends on the differential pressure between the water and oil-surfactant phases, with higher relative water pressures driving the formation of increasingly complex droplet arrays (Fig. 3).

The diverse pattern formation found in the rounded channels can be classified as follows. When the oil pressure greatly exceeds the water pressure, the water stream is held in check by surface tension and only the oil flows. As the water pressure is increased past a critical point, single monodisperse separated droplets are formed at a frequency of $20-80 \mathrm{~Hz}$. Small adjustments in the water pressure in this range change the radii of the formed droplets, with higher water pressures generating larger droplets. When the relative oil and water pressures are approximately balanced $\left(P_{w} \sim P_{o}\right)$, droplets are formed in a pearlnecklacelike configuration [Figs. 3(D) and 3(E)]. They stack up against each other during the transition from the $30 \mu \mathrm{m}$ channel to the wider $60 \mu \mathrm{m}$ channel due in part to the increased drag of the necklace (which is larger than the separated monodisperse droplets). At water pressures that slightly exceed the oil pressure $\left(P_{w}>P_{o}\right)$, the packing density of the droplets in the $60 \mu \mathrm{m}$ channel increases. The first complex structure that emerges with increasing oil pressure is a transition from the pearl-necklace shape into a zigzag pattern of droplets [Fig. $3(\mathrm{G})]$. At moderately higher water pressures $(\sim 10 \%$ higher than the relative oil pressure), shear occurs at both the crossflow junction and the transition from the narrow to wide microchannel. Polydisperse and bidisperse motifs appear as helices and patterned multilayer ribbon structures. The patterns remain coherent as the arrayed droplets flow down the entire length of the channel from the breakpoint

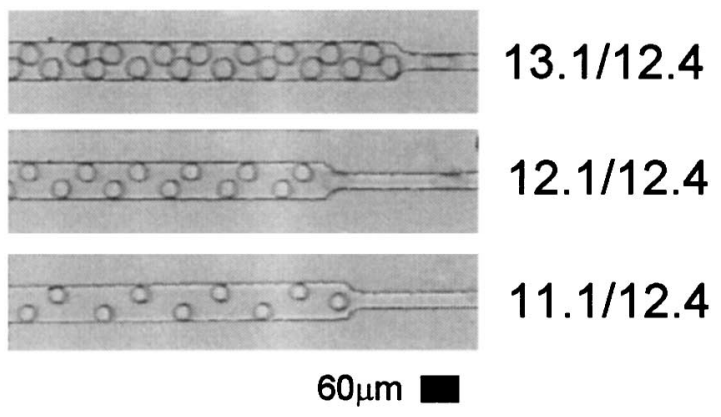

FIG. 2. Reverse micelles in square channels. Photomicrographs show the transition from the $30 \mu \mathrm{m}$ wide channel to the $60 \mu \mathrm{m}$ wide channel. Respective pressures for the water and oil/surfactant (hexadecane/2\% Span 80 ) are noted in the figure. 

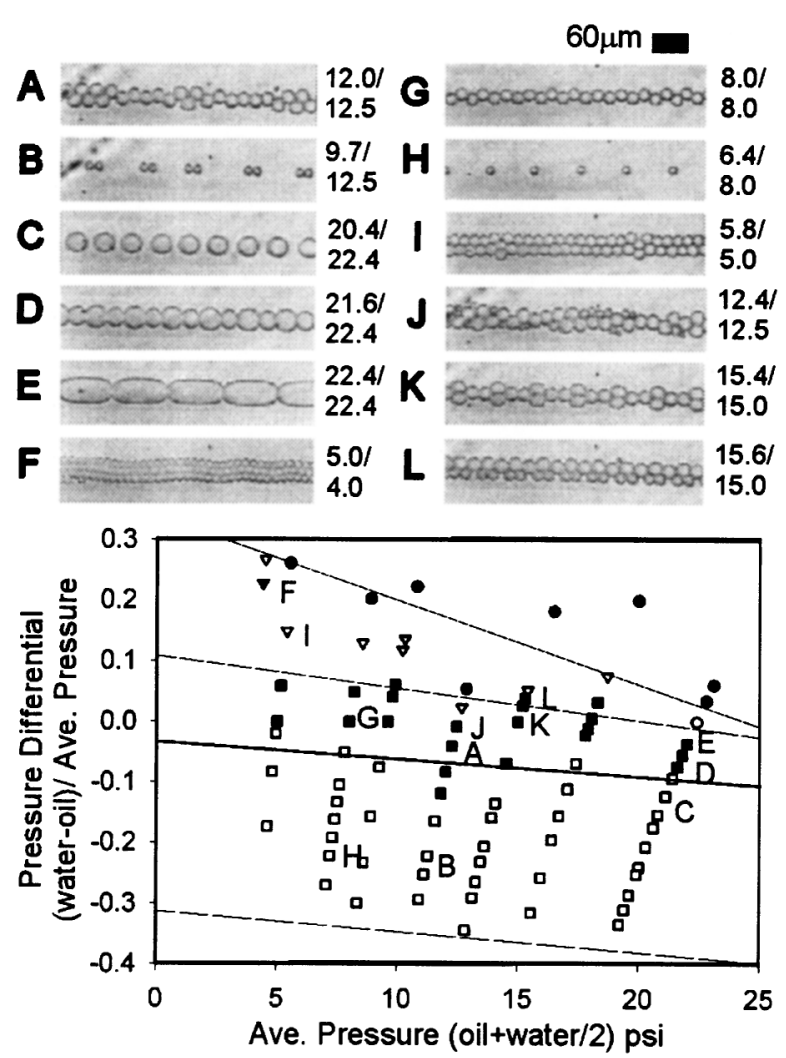

FIG. 3. Droplet patterns in rounded channels at different water and oil/surfactant pressures (noted in the figure) and the corresponding phase diagram depicting the relationship between the oil and water pressure differences and droplet morphology. Solid lines are used to define approximate boundaries between the following droplet states (top to bottom): solid water stream, ribbon layer, pearl necklace, single droplets, and solid oil stream. Symbol definition: solid water stream (solid circle); elongated droplets (open circle); triple droplet layer (solid triangle); double droplet layer (open triangle); jointed droplets (solid square); separated droplet (open square). Photomicrographs show $60 \mu \mathrm{m}$ channel regions downstream of the point of crossflow.

to the outlet $(\sim 4 \mathrm{~cm})$. At excessive water pressure, water coflows with the oil as separate streams, as one would expect for laminar flow of two conjoined streams.

The simplest model for droplet formation is based on the shear forces generated between the water and oil surfactant at the crossflow junction. The predicted size of a droplet under external shear force is approximated by equating the Laplace pressure with the shear force [13]:

$$
r \sim \frac{\sigma}{\eta \dot{\varepsilon}},
$$

where $r$ is the final droplet radius, $\sigma$ is the interfacial tension between the water/oil-surfactant, $\eta$ is the viscosity of the continuous phase, and $\dot{\varepsilon}$ is the shear rate.

In the microfluidic device, a shear gradient is established as water tries to expand into the pressurized continuous phase. The water stream never completely blocks the flow of the continuous phase, and the oil surfactant flows through the restriction at velocities up to $\sim 6.4 \mathrm{~cm} / \mathrm{s}$. Equation (1) gives a good approximation of the droplet sizes generated in the microfluidic device when the shear rate is estimated as $\dot{\varepsilon} \sim 2 v / y_{0}$, where $y_{0}$ is the channel radius at the center of flow estimated by triangular approximation, and $v$ is the velocity of the fluid through the gap. Predicted droplet sizes are within a factor of 2 of actual droplet size measured by video microscopy for monodisperse droplets generated at water and oil pressures ranging from 8.0-22.4 psi (Fig. 4).

Pattern formation in the microchannels appears to be driven by the drag force of the droplets and contain friction with the floor and ceiling of the device. As the droplets transition from the narrow crossflow junction to the $60 \mu \mathrm{m}$ channel, they slow down significantly relative to the oil phase. At higher droplet frequencies, they begin to collide, stacking up into organized patterns at the transition between the 30-60 $\mu \mathrm{m}$ channel. Complex structures form in rounded channels at high relative water pressures as colliding droplets are pushed from the center of the flow stream. The pattern formation results as a trade off between the interfacial tension of the droplets and the shape of the channels - droplets prefer to stay in the middle of the rounded channels in order not to pay an energy penalty for deformation in the crevices at the edge of the channels. Secondary shearing at the slowing junction also affects pattern formation - if the initial droplet is not commensurate with the size selected by the junction, then size dispersity is introduced to the stream and asymmetric motifs appear [Figs. 3(J) and 3(L)].

We have mapped a crude phase diagram that shows the pattern morphology is predominantly dependent on only the dimensionless differential input pressure. However, some of the most interesting patterns are found only at certain absolute values of the input pressure (Fig. 3). Somewhat surprisingly, these structures maintain a high degree

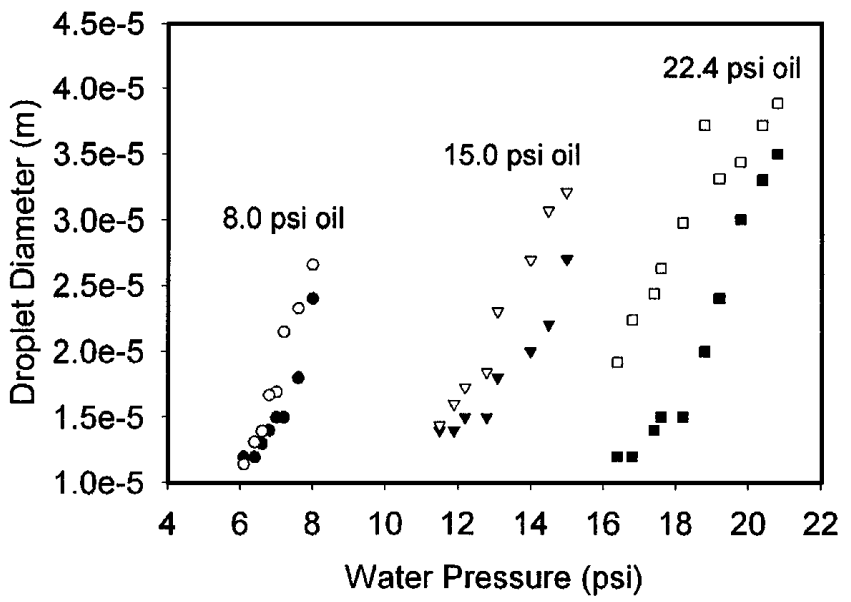

FIG. 4. Predicted vs actual drop size at different water and oil/surfactant pressures. The predicted sizes were calculated using Eq. (1). Open symbols, predicted size; solid symbols, experimental. 
of coherence, despite the fact that they are formed dynamically when the system is far from thermodynamic equilibrium. Furthermore, this system shows an unusual richness in the variety of phases it can display, especially considering that the boundary conditions (which also function as order parameters) are simply constant pressure applied to fluid inputs.

Pattern formation also occurs in granular materials, which have some striking similarities with our system. In both cases, the fundamental particles are so large that thermal fluctuations are negligible. Also, granular systems can display a "jamming" phenomena, in which the particles get trapped in metastable configurations that are difficult to escape from. The pearl necklaces and zigzag patterns in our system shown an ability to get into jammed states of high stress. The joined droplets behave similar to a spring, continually trying to relieve the added strain within the system by trying to orient themselves in the center of the stream. This behavior is shown by multiparticle defects that propagate as waves through the pearl necklaces with a speed greater than the droplet stream [Fig. 3(A)].

In conclusion, we have shown how instability can develop as a competition between shear forces and surface tension in a microfluidic device. The system is technically at low Reynolds number, but the equations of motion are nonlinear because the boundary between the two fluids is not static. Although we have outlined some of the basic physics leading to the vesicle forming instability and subsequent pattern formation, it is clear that more work needs to be done to achieve a complete understanding of the system. Since geometric effects play a significant role in the pattern formation, one should be able to take advantage of the powerful microfabrication technology both to explore the consequences of this observation and to provide stringent tests of theoretical models. This system may also find application as a component in a microfluidic screening chip, since it has been shown that subnanoliter vesicles have significant potential as tools for screening of biological and synthetic compounds [14-16].

We thank R. Goldstein for helpful discussions. This work was partially supported by Research Corporation and the NSF.

[1] M. C. Cross and P. C. Hohenberg, Rev. Mod. Phys. 65, 851 (1993).

[2] J.P. Gollub and J. S. Langer, Rev. Mod. Phys. 71, S396 (1999).

[3] For two interesting exceptions, see R.H. Liu et al., J. Microelectromech. Syst. 9, 190 (2000); D. Bokenkamp et al., Anal. Chem. 70, 232 (1998).

[4] J.P. Brody, P. Yager, R.E. Goldstein, and R.H. Austin, Biophys. J. 71, 3430 (1996).

[5] G. M. Whitesides and A. D. Stroock, Phys. Today (to be published).

[6] G. Mason, Curr. Opin. Colloid Interface Sci. 4, 231 (1999).

[7] P. B. Umbanhowar, V. Prasad, and D. A. Weitz, Langmuir 16, 347 (2000).

[8] T. Kawakatsu et al., J. Surf. Deterg. 3, 295-302 (2000).

[9] T. G. Mason and J. Bibette, Langmuir 13, 4600 (1997).

[10] T. Nakashima and M. Shimizu, Kagaku Kogaku Ronbunshu 19, 984 (1993).

[11] S. J. Peng and R. A. Williams, Chem. Eng. Res. Des. 76, 894 (1998).

[12] L. D. Landau and E. M. Lifshitz, Fluid Mechanics (Pergamon, London, 1959), 1 st ed.

[13] G. I. Taylor, Proc. R. Soc. London A 146, 501 (1934).

[14] D. T. Chiu et al., Science 283, 1892 (1999).

[15] D. S. Tawfik and A.D. Griffiths, Nature Biotech. 16, 652 (1998).

[16] C.-Y. Kung, M. D. Barnes, N. Lermer, W. B. Whitten, and J. M. Ramsey, Anal. Chem. 70, 658 (1998). 